\title{
Analytical expressions for spectral dependences of silver, gold, copper and aluminum dielectric permittivity
}

\author{
VOLODYMyr FITIO ${ }^{1}$, IRYNa YAREMCHUK ${ }^{1 *}$, OLEKSANDR VERNYHOR ${ }^{1}$, YAROSLAV BOBITSKI $^{1,2}$ \\ ${ }^{1}$ Lviv Polytechnic National University, Department of Photonics, \\ 12 S. Bandera St., 79013 Lviv, Ukraine \\ ${ }^{2}$ University of Rzeszów, College of Natural Sciences, \\ 1 Pigonia St., 35-959 Rzeszów, Poland \\ ${ }^{*}$ Corresponding author: iryna.y.yaremchuk@1pnu.ua
}

\begin{abstract}
In this work, the analytical expressions describing experimental data of silver, gold, copper and aluminum dielectric permittivity in a wide spectral range are presented. A comparison of samples production techniques, the measurement methods and the experimental data of different authors led to the conclusion that the most valid data are given by MCPEAK et al. (ACS Photonics 2(3), 2015, pp. 326-333) and BABAR et al. (Appl. Opt. 54(3), 2015, pp. 477-481), which are close to each other. Thus, the analytical expressions for silver, gold, copper and aluminum dielectric permittivity spectral dependences are based on it. The spectral range in which the dielectric permittivity is represented by the corresponding analytical expression is divided into several intervals. There is a specific function for each wavelength range.
\end{abstract}

Keywords: silver, gold, copper, aluminum, dielectric permittivity, Heaviside function.

\section{Introduction}

At present, there is a strong interest in noble metals based micro- and nanostructures. The most often it is silver, gold, copper and aluminum. The micro- and nanostructures have wide practical applications and as a result, the computer numerical experiments of the interaction of electromagnetic radiation with metal nanoparticles [ $\underline{1}-\underline{3}]$, as well as micro- and nanograting, which contain metals, are carried out [4-7]. However, the fabrication of the periodic nanostructures is rather complicated technologically. Whereas, computer simulation can predict the properties of such structures and find their optimal parameters for the appearance of one or another effect. The micro- and nanostructures which include the above listed metals can be manufactured based on the simulation data and carry out the real experiments.

Computer simulations are most often used for calculations of spectral dependences of reflection, transmission and absorption coefficients for gratings structures [ $\underline{5}, \underline{6}]$, and the absorption and scattering cross-sections for metallic nanoparticles [ $\underline{1}, \underline{2}]$. It has been shown experimentally that the resonance of localized surface plasmons in aluminum 
nanoparticles is observed in the ultraviolet region $[\underline{2}, \underline{8}]$, the plasmon resonance is detected approximately at the wavelength of $0.42 \mu \mathrm{m}$ in case of silver nanoparticles [ $\underline{3}$ ], at the wavelength of $0.52 \mu \mathrm{m}$ [9] in case of gold nanoparticles, and plasmon resonance of copper nanoparticless is shifted to the long wave region, and appears about $0.65 \mu \mathrm{m}[\underline{10}]$.

The spectral region of the resonance of localized surface plasmons in nanoparticles depends on the metal, size and local dielectric environment. However, the resonance spectral shift of metal nanoparticles is not so huge in comparison to the resonance spectral shift of metal nanograting. However, the resonance in the dielectric slit of metal grating $[11,12]$, the surface plasmon-polariton resonance $[\underline{5}, \underline{13}]$ and the waveguide modes resonance [6] are observed in a wide spectral range. Moreover, the position of the resonance peak depends on the parameter of the periodic nanostructure and can be tuned in the wide region $[\underline{11}, \underline{13}]$.

In order to accurately carry out computer simulations of the interaction of electromagnetic waves with nanostructures, it is reasonable to have spectral dependences of dielectric permittivities of noble metals in the analytical form. Nowadays, a number of experimental dependences of dielectric permittivity of metals on the wavelength are known which are represented in works [14-26]. Theoretical Brendel-Bormann model and Lorentz-Drude model were published in 1998 by RaKić et al. [27]. These models summarized the experimental results of different authors, which were obtained before 1998 and were collected in the book [26]. Obviously, these models did not well correlate with the experiments data obtained recently. Moreover, the experimental spectral dependences of dielectric constants, measured by different authors at different periods of time, for example, for silver [14, 26], are quite different. This can be explained by the fact that different starting materials of different purity, different fabrication techniques and various measurement methods that were available at the time of research from the sixties of the last century [26] to 2017 [25] have been used. The mathematical expressions for real and imaginary parts of the silver dielectric permittivity in the range of wavelengths from 0.4 to $2 \mu \mathrm{m}$ [27] have been presented on the basis of data [18]. These expressions are used in many studies $[\underline{5}, \underline{12}, \underline{28}]$. However, these data are measured with low precision, as noted in work [18], and the lower limit of the chosen analytical expression of $0.4 \mu \mathrm{m}$ is insufficient to study the resonance phenomena in silver nanoparticles or silver-based nanograting structures. Different measurement data and theoretical models can be found at https://refractiveindex.info. Our study of the site data for gold, silver, copper and aluminum, and related publications regarding preparation samples and measurements showed that the most validate data of the spectral dependences of dielectric permittivity are given in $[14, \underline{15}]$ for the first three metals and works $[14,17]$ for aluminum. The researches described in [14] were carried out with sufficient pure metals: $99.99 \%$ for $\mathrm{Ag}, 99.99 \%$ for $\mathrm{Cu}, 99.999 \%$ for $\mathrm{Au}$, and $99.999 \%$ for Al. The results of experiments of these two groups of scientists $[14,15]$ practically agree in the wide spectral range from 0.3 to $1.7 \mu \mathrm{m}$. Therefore, in present work the results of $[\underline{14}, \underline{15}]$ were used as a base for mathematical expressions describing the dielectric permittivity of $\mathrm{Ag}, \mathrm{Au}$ in the spectral range from 0.29 to $2.0 \mu \mathrm{m}$. In case of copper, the analytical expressions are presented in the range of wavelengths 
from 0.4 to $4.2 \mu \mathrm{m}$ on the base of papers [14, 15]. Analytical expressions for aluminum are found from experimental data of works $[\underline{14}, 17]$ and are valid in the range of wavelengths from 0.1 to $2 \mu \mathrm{m}$.

\section{Spectral dependences of the metals dielectric permittivity in the analytical form}

In the first step, the experimental data [14-26] and theoretical models [27] of silver dielectric permittivity have been analyzed (see Fig. 1). One can conclude from Fig. 1

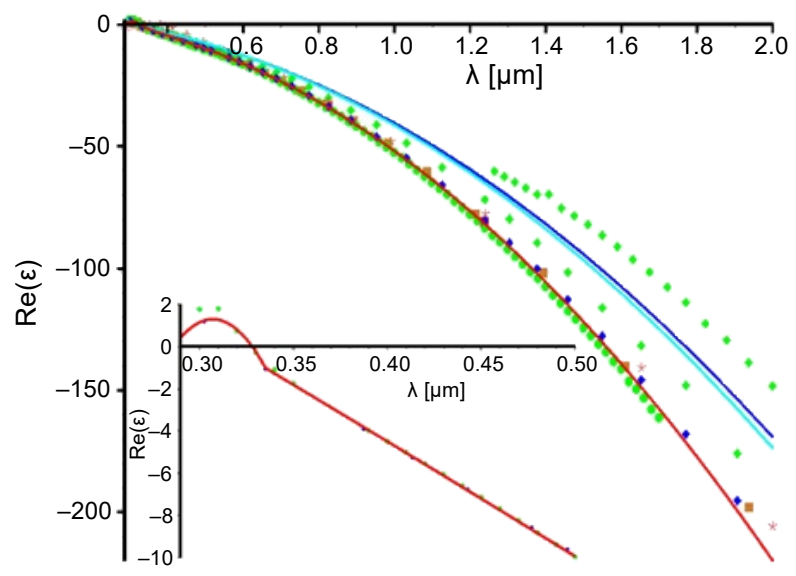

a

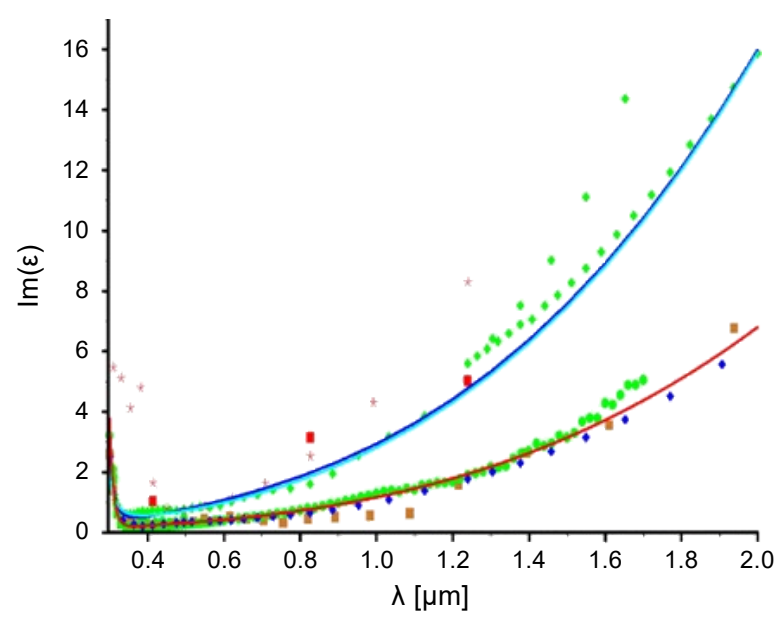

b

Fig. 1. Spectral dependences of the real (a) and imaginary (b) parts of silver dielectric permittivity on wavelength, where the green circles are data from [14], the dark blue rhombs are data from [15], the green rhombs are data from [26], the brown squares are data from [18], the red squares are data from [17], the brown stars are data from [리], the dark blue curves is the Brendel-Bormann model from [27], the blue curves is the Lorentz-Drude model from [27], the red curves are data calculated by obtained analytical expressions (1a) and (1b). Inset in part (a) shows spectral dependence of the real part of silver dielectric permittivity in the wavelength range from 0.29 to $0.5 \mu \mathrm{m}$ according to data from $[14, \underline{15}]$ and Eq. (1a). 
that the experimental data presented in $[\underline{14}, \underline{15}]$ are very close to each other, and there is no significant experimental data jumps with changing the wavelength. It should also be noted that the experimental points from work [18] published in 1972 are very close to the data used from $[14,15]$. However, jumps in the dependence of silver dielectric permittivity on the wavelength are observed, especially for the imaginary part of dielectric permittivity. The analytical dependences were determined on the base of data $[14,15]$, for which the corresponding curves (in Fig. 1, continuous curves in red color) are the best fitted with experimental data in the spectral range from 0.29 to $2.066 \mu \mathrm{m}$. However, one relatively simple function cannot represent the spectral dependence of silver dielectric permittivity in the given wavelengths range. Therefore, the total wavelengths range was divided into three intervals: [0.29 $\mu \mathrm{m}, 0.335 \mu \mathrm{m}]$, $[0.335 \mu \mathrm{m}, 0.521 \mu \mathrm{m}],[0.521 \mu \mathrm{m}, 2.066 \mu \mathrm{m}]$, respectively, for the real part of dielectric permittivity. The individual function was selected for each interval as follows: $r_{1}(\lambda)=$ $=1.3-2950(\lambda-0.307)^{2}, r_{2}(\lambda)=16.3-50 \lambda-5 \lambda^{2}$, and $r_{3}(\lambda)=4.093-56 \lambda^{2}$.

In order to represent the dielectric permittivity by certain functions only in the corresponding interval, the Heaviside function

$$
H(x)=\left\{\begin{array}{lll}
1, & \text { if } & x>0 \\
\text { undefined, } & \text { if } & x=0 \\
0, & \text { if } & x<0
\end{array}\right.
$$

can be used.

The impulse function with amplitude 1 and the width corresponding to the certain interval $\Pi(\lambda)=[H(\lambda-0.29)-H(\lambda-0.335)]$ can be constructed on the base of the Heaviside function. Then the real part of silver dielectric permittivity in the range from 0.29 to $2.066 \mu \mathrm{m}$ can be written as follows:

$$
\begin{aligned}
\operatorname{Re}(\varepsilon)= & r_{1}(\lambda)[H(\lambda-0.29)-H(\lambda-0.335)] \\
& +r_{2}(\lambda)[H(\lambda-0.335)-H(\lambda-0.521)] \\
& +r_{3}(\lambda)[H(\lambda-0.521)-H(\lambda-2.066)]
\end{aligned}
$$

The imaginary part of silver dielectric permittivity in the range from 0.29 to $2.066 \mu \mathrm{m}$ can be described by only one analytic function as follows:

$$
\operatorname{Im}(\varepsilon)=0.03+0.36 \lambda+0.72 \lambda^{3}+3 \exp [-100(\lambda-0.299)]
$$

It can be seen that the experimental data of works $[\underline{14}, \underline{15}]$ are very close together, especially for the real part of dielectric permittivity, and the continuous red curve according to (1) is between the experimental points of these works. It should also be noted that the experimental points for the imaginary part of the dielectric permittivity of works $[14,15]$ have greater differences in comparison with the experimental points of 

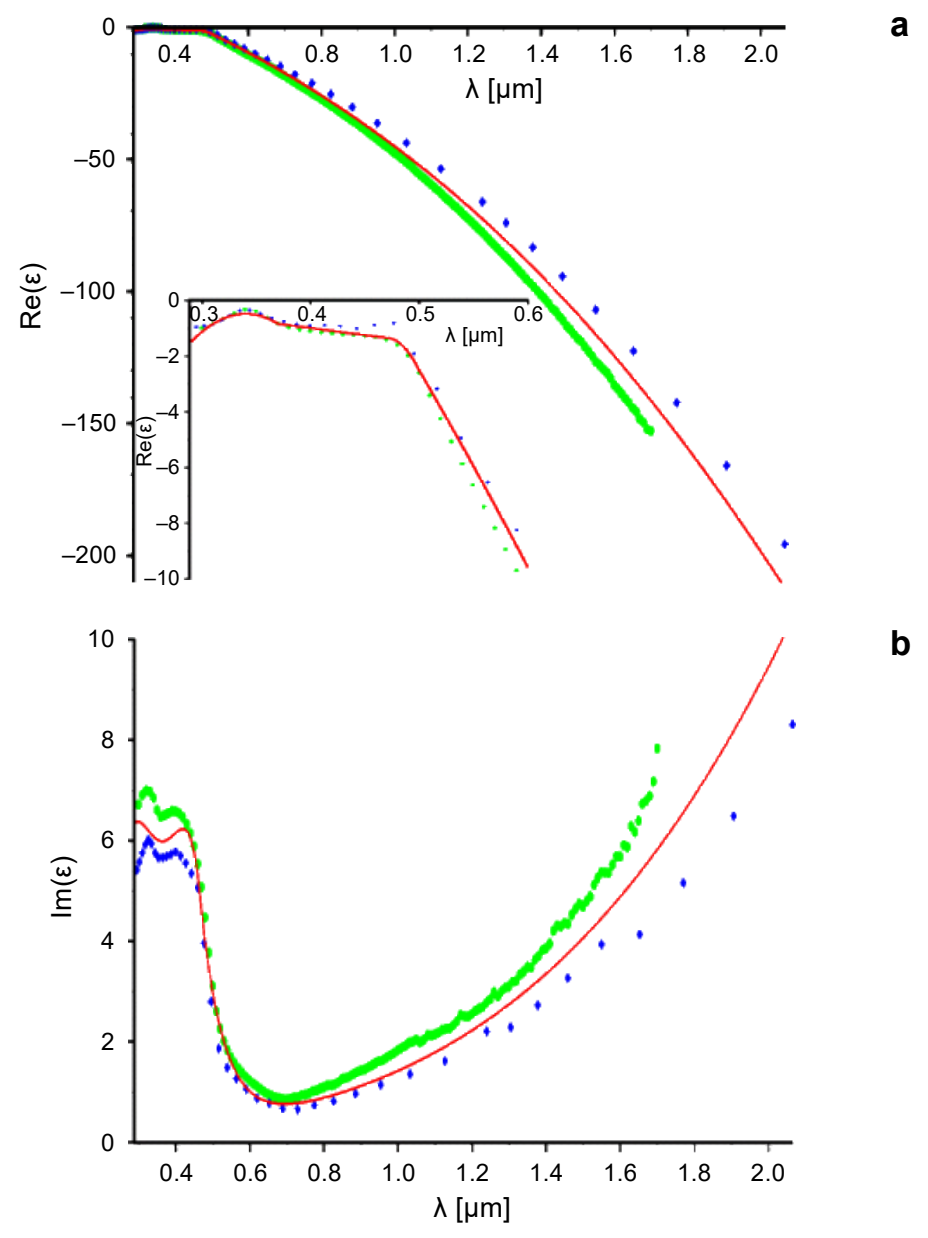

Fig. 2. Spectral dependences of real (a) and imaginary (b) parts of gold dielectric permittivity on wavelength, where the green circles are data from [14], the blue rhombs are data from [15], red curves are data calculated by obtained analytical expressions (2a) and (2b). Inset in part (a) shows the spectral dependence of the real part of gold dielectric permittivity in the wavelength range from 0.29 to $0.6 \mu \mathrm{m}$ according to data from $[14,15]$ and Eq. (2a).

the real part of the dielectric permittivity. The same situation is observed for copper and gold.

The corresponding curves have been obtained on the base of works [ $[\underline{14} \underline{20}, \underline{24}, \underline{26}, \underline{27}]$ for real and imaginary parts of gold dielectric permittivity. However, only experimental points on the base of works $[\underline{14}, \underline{15}]$, and the continuous curve on the base of analytical expressions (2a) and (2b) are given in Fig. 2. The real part of the spectral dependence of gold dielectric permittivity over the total wavelength range can be represented by four functions in the form of polynomials as follows:

$$
r_{1}(\lambda)=-0.485-400(\lambda-0.34)^{2}
$$




$$
\begin{aligned}
& r_{2}(\lambda)=-1.0955-5(\lambda-0.42)-4.9(\lambda-0.42)^{3} \\
& r_{3}(\lambda)=-1.346-1300(\lambda-0.47)^{2} \\
& r_{4}(\lambda)=-2.516-67(\lambda-0.5)-35(\lambda-0.5)^{2}-4.9(\lambda-0.5)^{4}+0.7(\lambda-0.5)^{6}
\end{aligned}
$$

As a result, the real part of gold dielectric permittivity can be written using the Heaviside function as follows:

$$
\begin{aligned}
\operatorname{Re}(\varepsilon)= & r_{1}(\lambda)[H(\lambda-0.29)-H(\lambda-0.37)] \\
& +r_{2}(\lambda)[H(\lambda-0.37)-H(\lambda-0.47)] \\
& +r_{3}(\lambda)[H(\lambda-0.47)-H(\lambda-0.5)] \\
& +r_{4}(\lambda)[H(\lambda-0.5)-H(\lambda-2.066)]
\end{aligned}
$$

It is enough to use the two functions to describe the imaginary part of gold dielectric permittivity:

$$
\begin{aligned}
g_{1}(\lambda)= & 5.99-1.2(\lambda-0.36)+170(\lambda-0.36)^{2}-23000(\lambda-0.36)^{4} \\
g_{2}(\lambda)= & 0.27+1.4(\lambda-0.46)+0.5(\lambda-0.46)^{2}+1.6(\lambda-0.46)^{3} \\
& +5 \exp [-16(\lambda-0.46)]
\end{aligned}
$$

Finally, the spectral dependence of the imaginary part of gold dielectric permittivity can be written as follows:

$$
\begin{aligned}
\operatorname{Im}(\varepsilon)= & g_{1}(\lambda)[H(\lambda-0.29)-H(\lambda-0.46)] \\
& +g_{2}(\lambda)[H(\lambda-0.46)-H(\lambda-2.066)]
\end{aligned}
$$

The spectral dependences of gold dielectric permittivity are shown in Fig. 2. It can be seen that the disagreement in the experimental data of works [14] and [1] for gold is more pronounced than for silver.

Recently, the interest in copper nanoparticles has increased, for which the localized surface plasmon resonance is shifted to the long-wave region of the spectrum. The corresponding curves are obtained on the base of work $[\underline{14}-18, \underline{20}$, 27] for real and imaginary parts of copper dielectric permittivity. However, only the experimental points 


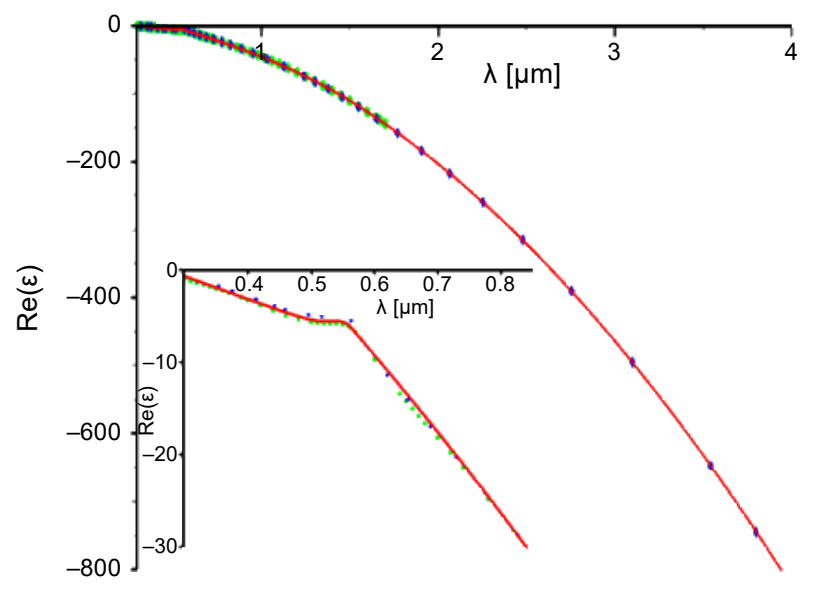

a

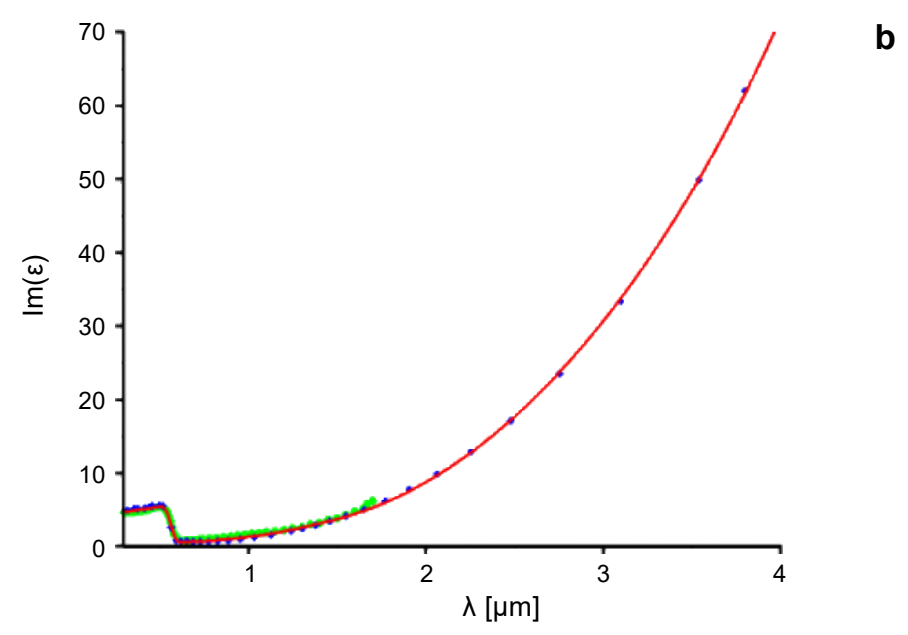

Fig. 3. Spectral dependences of the real (a) and imaginary (b) parts of copper dielectric permittivity, where the green circles are data from [14], the dark blue rhombs are data from [15], the red curves are data calculated by obtained analytical expressions (3a) and (3b). Inset in part (a) shows the spectral dependence of the real part of copper dielectric permittivity in the wavelength range from 0.3 to $0.85 \mu \mathrm{m}$ according to data from $[\underline{14}, \underline{15}]$ and Eq. (3a).

on the base of works $[\underline{14}, \underline{15}]$, as well as a continuous curve based on analytical expressions (3a) and (3b), are presented in Fig. 3. The total wavelength range is divided into three intervals for the real part of copper dielectric permittivity as follows:

$$
r_{1}(\lambda)=-0.7-25(\lambda-0.3)+\frac{1.3}{1+100\left(\frac{1}{0.55}-\frac{1}{\lambda}\right)^{2}}
$$




$$
\begin{aligned}
& r_{2}(\lambda)=-6.024-80(\lambda-0.56)-15(\lambda-0.56)^{2}-19(\lambda-0.56)^{3} \\
& r_{3}(\lambda)=-135.65-176(\lambda-1.65)-50(\lambda-1.65)^{2}
\end{aligned}
$$

Thus, the real part of the spectral dependence of copper dielectric permittivity can be written as follows:

$$
\begin{aligned}
\operatorname{Re}(\varepsilon)= & r_{1}(\lambda)[H(\lambda-0.3)-H(\lambda-0.56)] \\
& +r_{2}(\lambda)[H(\lambda-0.56)-H(\lambda-1.65)] \\
& +r_{3}(\lambda)[H(\lambda-1.65)-H(\lambda-4.2)]
\end{aligned}
$$

In case of the imaginary part of copper dielectric permittivity, the total wavelength range is divided into four intervals as follows:

$$
\begin{aligned}
g_{1}(\lambda)= & 4.7+3.5(\lambda-0.3) \\
g_{2}(\lambda)= & 3-\frac{2.99 \tanh [30(x-0.56)]}{1+50(x-0.56)^{2}} \\
g_{3}(\lambda)= & 0.59+0.74(\lambda-0.62)+2.9(\lambda-0.62)^{2}+0.6(\lambda-0.62)^{4} \\
g_{4}(\lambda)= & 3.2182+4.9(\lambda-1.41)+7.6(\lambda-1.41)^{2}+0.05(\lambda-1.41)^{4} \\
& +0.009(\lambda-1.41)^{6}
\end{aligned}
$$

Therefore, the imaginary part of copper dielectric permittivity can be written as follows:

$$
\begin{aligned}
\operatorname{Im}(\varepsilon)= & g_{1}(\lambda)[H(\lambda-0.3)-H(\lambda-0.5)] \\
& +g_{2}(\lambda)[H(\lambda-0.5)-H(\lambda-0.62)] \\
& +g_{3}(\lambda)[H(\lambda-0.62)-H(\lambda-1.41)] \\
& +g_{4}(\lambda)[H(\lambda-1.41)-H(\lambda-4.2)]
\end{aligned}
$$

The spectral dependences of copper dielectric permittivity are shown in Fig. 3. As follows from this figure, the experimental points are fitted with the continuous curve rather accurately. Moreover, it was necessary to use more complex functions, and not just polynomials, for the exact representation of spectral dependences. 
The localized surface plasmon resonance in aluminum nanoparticles occurs in the ultraviolet spectrum region [2]. Now it is a very promising material for wide application, for example, a microlaser based on the plasmonic effect in aluminum was fabricated in the ultraviolet region of the spectrum with a low threshold [ $\underline{8}]$. The spectral dependence of the imaginary part of aluminum dielectric permittivity is characterized by the clear local maximum at the wavelength of $0.805 \mu \mathrm{m}[\underline{14}, \underline{16}, \underline{17}, \underline{27}, \underline{29}]$. As a result, there are some difficulties in the search of the analytical dependences.

The corresponding curves on the base of the works $[\underline{14}, \underline{16}, \underline{17}, \underline{27}, \underline{29}]$ were obtained for the real and imaginary parts of aluminum dielectric permittivity. Figure 4

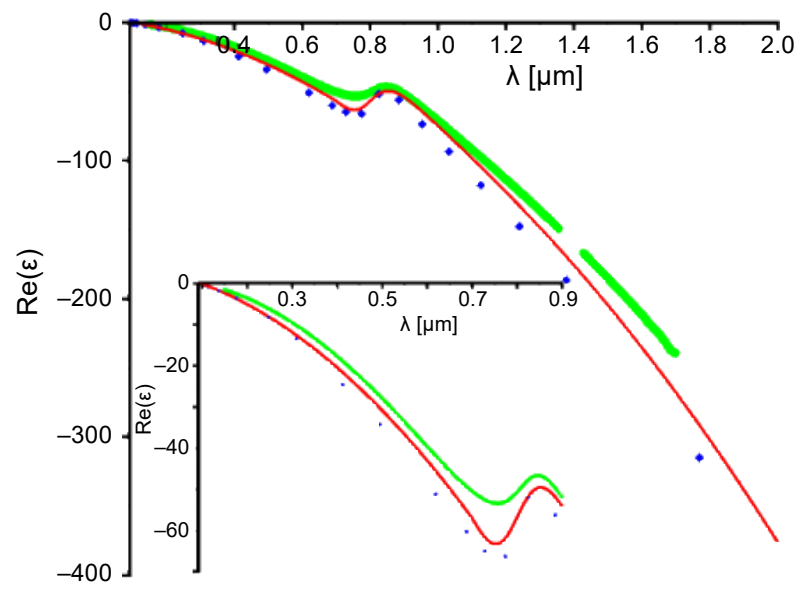

a

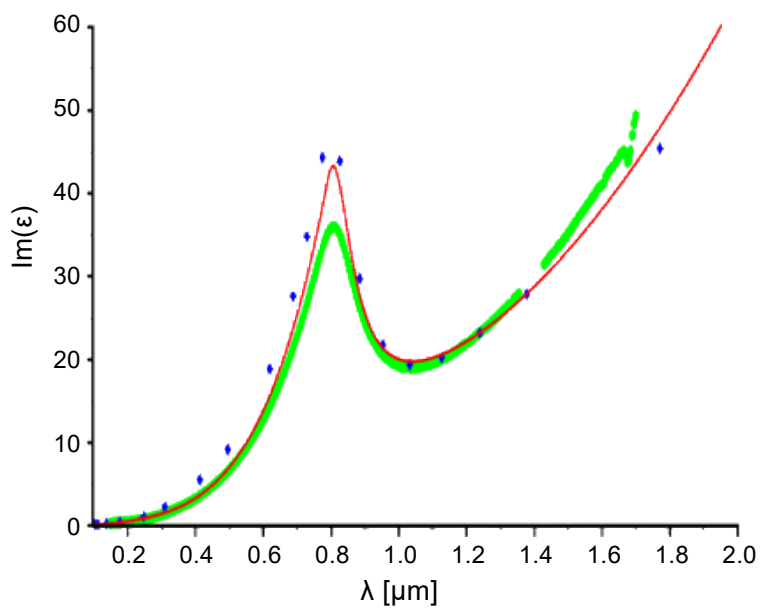

b

Fig. 4. Spectral dependences of the real (a) and imaginary (b) parts of aluminum dielectric permittivity, where the green circles are data from [14], the dark blue rhombs are data from [17], red curves are data calculated by obtained analytical expressions (4a) and (4b). Inset in part (a) shows the spectral dependence of the real part of aluminum dielectric permittivity in the wavelength range from 0.3 to $0.9 \mu \mathrm{m}$ according to the data from $[\underline{14}, \underline{17}]$ and Eq. (4a). 
shows the experimental points based on the works $[14, \underline{17}]$ as well as the continuous curve based on analytical expressions (4a) and (4b). The real part of the spectral dependence of aluminum dielectric permittivity in the range from 0.1 to $2.0 \mu \mathrm{m}$ can be described by two of the following functions:

$$
\begin{aligned}
& r_{1}(\lambda)=0.1-40(\lambda-0.09537)-90(\lambda-0.09537)^{2} \\
& r_{2}(\lambda)=-39.963-140(\lambda-0.7)-95(\lambda-0.7)^{2}-\frac{250\left(\frac{1}{\lambda}-\frac{1}{0.805}\right)}{1+50\left(\frac{1}{\lambda}-\frac{1}{0.805}\right)^{2}}
\end{aligned}
$$

As a result, the real part of the aluminum dielectric permittivity can be written as follows:

$$
\begin{aligned}
\operatorname{Re}(\varepsilon)= & r_{1}(\lambda)[H(\lambda-0.09537)-H(\lambda-0.7)] \\
& +r_{2}(\lambda)[H(\lambda-0.7)-H(\lambda-2.0)]
\end{aligned}
$$

The imaginary part of the spectral dependence of aluminum dielectric permittivity can be described in the wavelength range from 0.1 to $2.0 \mu \mathrm{m}$ by the following two functions:

$$
\begin{aligned}
g_{1}(\lambda)= & 0.0771+3(\lambda-0.09537)+12(\lambda-0.09537)^{2} \\
& +140(\lambda-0.09537)^{4}+10(\lambda-0.09537)^{6} \\
g_{2}(\lambda)= & 12.122+8.1(\lambda-0.7869)+28(\lambda-0.7869)^{2}+\frac{31}{1+90\left(\frac{1}{\lambda}-\frac{1}{0.805}\right)^{2}}
\end{aligned}
$$

Therefore, the imaginary part of aluminum dielectric permittivity depending on the wavelength, can be written as

$$
\begin{aligned}
\operatorname{Im}(\varepsilon)= & g_{1}(\lambda)[H(\lambda-0.09537)-H(\lambda-0.7869)] \\
& +g_{2}(\lambda)[H(\lambda-0.7869)-H(\lambda-2.0)]
\end{aligned}
$$

The spectral dependences of aluminum dielectric permittivity in the wavelength range from 0.1 to $2.0 \mu \mathrm{m}$ are presented in Fig. 4.

The data of the silver dielectric permittivity from work [22] published in 2015, and data according to the analytical Eqs. (1a) and (1b) are given in Table 1, for the some wavelengths. 
T a b l e 1. The silver dielectric permittivity.

\begin{tabular}{|c|c|c|c|c|c|c|c|c|c|}
\hline \multirow{2}{*}{$\lambda[\mu \mathrm{m}]$} & \multicolumn{2}{|l|}{$\operatorname{Re}(\varepsilon)$} & \multicolumn{2}{|l|}{$\operatorname{Im}(\varepsilon)$} & \multirow{2}{*}{$\lambda[\mu \mathrm{m}]$} & \multicolumn{2}{|l|}{$\operatorname{Re}(\varepsilon)$} & \multicolumn{2}{|l|}{$\operatorname{Im}(\varepsilon)$} \\
\hline & Ref. [22] & Eq. (1a) & Ref. [22] & Eq. (1b) & & Ref. [22] & Eq. (1a) & Ref. [2] & Eq. (1b) \\
\hline$\overline{0.3}$ & 1.756 & 1.155 & 2.937 & 2.872 & 1.2 & -70.082 & -76.547 & 2.654 & 1.706 \\
\hline 0.4 & -4.369 & -4.50 & 0.243 & 0.220 & 1.3 & -82.758 & -90.547 & 3.354 & 2.080 \\
\hline 0.5 & -9.378 & -9.95 & 0.317 & 0.300 & 1.4 & -96.387 & -105.67 & 4.168 & 2.510 \\
\hline 0.6 & -15.127 & -16.067 & 0.438 & 0.402 & 1.5 & -111.03 & -121.91 & 5.110 & 3.000 \\
\hline 0.7 & -21.802 & -23.347 & 0.616 & 0.529 & 1.6 & -126.71 & 139.27 & 6.186 & 3.555 \\
\hline 0.8 & -29.465 & -31.747 & 0.859 & 0.687 & 1.7 & -143.42 & -157.75 & 7.406 & 4.179 \\
\hline 0.9 & -38.118 & -41.267 & 1.176 & 0.879 & 1.8 & -161.17 & -177.35 & 8.781 & 4.877 \\
\hline 1.0 & -47.773 & -51.907 & 1.575 & 1.110 & 1.9 & -179.95 & 198.07 & 10.315 & 5.652 \\
\hline 1.1 & -58.428 & -63.667 & 2.066 & 1.384 & 2.0 & -199.48 & 219.91 & 12.013 & 6.510 \\
\hline
\end{tabular}

T a b l e 2. The gold dielectric permittivity.

\begin{tabular}{|c|c|c|c|c|c|c|c|c|c|}
\hline \multirow{2}{*}{$\lambda[\mu \mathrm{m}]$} & \multicolumn{2}{|l|}{$\operatorname{Re}(\varepsilon)$} & \multicolumn{2}{|l|}{$\operatorname{Im}(\varepsilon)$} & \multirow{2}{*}{$\lambda[\mu \mathrm{m}]$} & \multicolumn{2}{|l|}{$\operatorname{Re}(\varepsilon)$} & \multicolumn{2}{|l|}{$\operatorname{Im}(\varepsilon)$} \\
\hline & Ref. [25] & Eq. (1a) & Ref. [25] & Eq. (1b) & & Ref. [25] & Eq. (1a) & Ref. [25] & Eq. (1b) \\
\hline$\overline{0.3}$ & -1.13 & -1.13 & 6.42 & 6.37 & 1.2 & -67.74 & -67.67 & 4.34 & 2.23 \\
\hline 0.4 & -1.27 & -1.00 & 6.37 & 6.15 & 1.3 & -80.82 & -80.34 & 5.53 & 2.75 \\
\hline 0.5 & -2.63 & -2.52 & 3.12 & 2.96 & 1.4 & -94.80 & -94.01 & 6.79 & 3.36 \\
\hline 0.6 & -9.99 & -9.57 & 1.29 & 1.01 & 1.5 & -109.7 & -108.7 & 7.96 & 4.07 \\
\hline 0.7 & -17.63 & -17.32 & 1.00 & 0.76 & 1.6 & -125.7 & -124.5 & 9.52 & 4.89 \\
\hline 0.8 & -25.93 & -25.81 & 1.29 & 0.89 & 1.7 & -142.8 & -141.4 & 10.98 & 5.83 \\
\hline 0.9 & -34.97 & -35.04 & 1.74 & 1.12 & 1.8 & -159.7 & -159.4 & 12.78 & 6.89 \\
\hline 1.0 & -44.99 & -45.06 & 2.27 & 1.42 & 1.9 & -178.7 & 178.5 & 14.84 & 8.10 \\
\hline 1.1 & -55.87 & -55.92 & 3.29 & 1.79 & 2.0 & -198.6 & 198.6 & 17.14 & 9.46 \\
\hline
\end{tabular}

A comparison of the data given in the columns 2 and 3 (Table 1) shows approximately $10 \%$ difference in the total spectral wavelengths range from 0.3 to $2.0 \mu \mathrm{m}$. However, the imaginary parts according to Eq. (1b) are almost twice less than in [22], with the exception of the interval from 0.3 to $0.7 \mu \mathrm{m}$.

The gold dielectric permittivity from work [25] for the film thickness of $117 \mathrm{~nm}$, published in 2017, and data according to the obtained analytical Eqs. (2a) and (2b) are given in Table 2, for some wavelengths. The comparison of the data presented in the columns 2 and 3 (Table 2) shows the good agreement in the spectral wavelength range from 0.3 to $2 \mu \mathrm{m}$.

However, the imaginary parts according to Eq. (2b) are almost twice lower than in [25], except for the interval from 0.3 to $0.8 \mu \mathrm{m}$. It seems that the imaginary part of dielectric permittivity is sensitive to the purity of the original metal, fabrication technology of metal films and film thickness. This conclusion agrees with other results of work [25], where gold dielectric permittivity is presented for film thicknesses of 25, 53 and $117 \mathrm{~nm}$, and with the results presented in Table 1. 


\section{Conclusion}

The corresponding analytical expressions for the dependence of dielectric permittivity on wavelength are determined based on experimental measurements of dielectric permittivity in the wavelength range from 0.3 to $2.0 \mu \mathrm{m}$ for silver and gold, from 0.3 to $4.2 \mu \mathrm{m}$ for copper and from 0.1 to $2 \mu \mathrm{m}$ for aluminum. Moreover, the total spectral range (with the exception of the imaginary part of silver dielectric permittivity) must be divided into several intervals. The corresponding function was selected for each interval of wavelengths, preferably in the form of polynomials. The selection of the function on each interval was carried out by the Heaviside function.

It should also be noted that the analytic function is continuous throughout the total spectral range. Such an analytical representation is very convenient to use in various calculations in the wide spectral range of the interaction of electromagnetic radiation with nanoparticles of the corresponding metals, or with periodic structures, which contain these metals. However, there is a limitation for using these analytical expressions. The dielectric permittivity will not be determined at the boundary of two adjacent intervals, since as defined, the Heaviside function is not defined at zero. Here the Heaviside function can be defined as $H(0)=1 / 2$, or avoid calculating the wavelength corresponding to the boundary between two adjacent intervals.

Acknowledgments - This research was funded by the Ministry of Education and Science of Ukraine (grants DB/Fotonika No. 0117 U007176 and DB/MEV No. 0118U000267).

\section{References}

[1] Maier S.A., Atwater H.A., Plasmonics: localization and guiding of electromagnetic energy in metal/ dielectric structures, Journal of Applied Physics 98(1), 2005, article 011101, DOI: 10.1063/1.1951057.

[2] Martin J., Proust J., GÉRARd D., Plain J., Localized surface plasmon resonances in the ultraviolet from large scale nanostructured aluminum films, Optical Materials Express 3(7), 2013, pp. 954-959, DOI: 10.1364/OME.3.000954.

[3] Bulavinets T., Yaremchuk I., Bobitski YA., Modeling optical characteristics of multilayer nanoparticles of different sizes for applications in biomedicine, [In] Nanophysics, Nanophotonics, Surface Studies, and Applications, [Eds.] O. Fesenko, L. Yatsenko, Springer Proceedings in Physics, Vol. 183. Springer, Cham, 2016, pp. 101-115, DOI: 10.1007/978-3-319-30737-4_9.

[4] Weismann M., Gallagher D.F.G., Panoiu N.C., Accurate near-field calculation in the rigorous coupled-wave analysis method, Journal of Optics 17(12), 2015, article 125612, DOI: 10.1088/2040-8978/ $17 / 12 / 125612$.

[5] ZINENKO T.L., MARCINIAK M., NOSICH A.I., Accurate analysis of light scattering and absorption by an infinite flat grating of thin silver nanostrips in free space using the method of analytical regularization, IEEE Journal of Selected Topics in Quantum Electronics 19(3), 2013, article 9000108, DOI: 10.1109/JSTQE.2012.2227685.

[6] Fitio V.M., Bobitski YA.V., Resonance effects in a dielectric grating; total absorption of electromagnetic waves by dielectric grating on metal system, Journal of Optics A: Pure and Applied Optics 6(10), 2004, pp. 943-951, DOI: 10.1088/1464-4258/6/10/004.

[7] Joye C.D., Calame J.P., CoOK A.M., Garven M., High-power copper gratings for a sheet-beam traveling-wave amplifier at G-band, IEEE Transactions on Electron Devices 60(1), 2013, pp. 506-509, DOI: $10.1109 /$ TED.2012.2226591. 
[8] Zhang Q., Li G., Liu X., Qian F., Li Y., Sum T.C., Lieber C.M., Xiong Q., A room temperature low-threshold ultraviolet plasmonic nanolaser, Nature Communications 5, 2014, article 4953, DOI: $10.1038 /$ ncomms5953.

[9] Eustis S., EL-SAYED M.A., Why gold nanoparticles are more precious than pretty gold: noble metal surface plasmon resonance and its enhancement of the radiative and nonradiative properties of nanocrystals of different shapes, Chemical Society Reviews 35(3), 2006, pp. 209-217, DOI: 10.1039/B514191E.

[10] LiU P., WANG H., Li X., Rui M., Zeng H., Localized surface plasmon resonance of Cu nanoparticles by laser ablation in liquid media, RSC Advances 5(97), 2015, pp. 79738-79745, DOI: 10.1039 / C5RA14933A.

[11] Stelle J.M., Moran C.E., Lee A., Aguirre C.M., Halas N.J., Metallodielectric gratings with subwavelength slots: optical properties, Physical Review B 68(20), 2003, article 205103, DOI: 10.1103/PhysRevB.68.205103.

[12] Fitio V.M., Transmissions of metallic gratings with narrow slots, Proc. 8th International Conference on Laser and Fiber-Optical Networks Modeling, Kharkiv, 2006, pp. 113-116.

[13] Fitio V.M., LABa H.P., BoBitski Ya.V., Absorption of electromagnetic waves into periodic structure and thin film of metal when a resonance of plasmons appears as a result of prism excitation, Telecommunications and Radio Engineering 66(7), 2007, pp. 607-618, DOI: 10.1615/TelecomRadEng. v66.i7.40.

[14] McPeak K.M., Jayanti S.V., Kress S.J.P., Mayer S., Iotti S., Rossinelli A., Norris D.J., Plasmonic films can easily be better: rules and recipe, ACS Photonics 2(3), 2015, pp. 326-333, DOI: $10.1021 /$ ph5004237.

[15] Babar S., Weaver J.H., Optical constants of Cu, Ag, and Au revisited, Applied Optics 54(3), 2015, pp. 477-481, DOI: 10.1364/AO.54.000477.

[16] Ordal M.A., Bell R.J., Alexander R.W., Long L.L., Querry M.R., Optical properties of fourteen metals in the infrared and far infrared: $\mathrm{Al}, \mathrm{Co}, \mathrm{Cu}, \mathrm{Au}, \mathrm{Fe}, \mathrm{Pb}, \mathrm{Mo}, \mathrm{Ni}, \mathrm{Pd}, \mathrm{Pt}, \mathrm{Ag}, \mathrm{Ti}, \mathrm{V}$, and $\mathrm{W}$, Applied Optics 24(24), 1985, 4493-4499, DOI: 10.1364/AO.24.004493.

[17] Hagemann H.-J., Gudat W., Kunz C., Optical constants from the far infrared to the $x$-ray region: $\mathrm{Mg}, \mathrm{Al}, \mathrm{Cu}, \mathrm{Ag}, \mathrm{Au}, \mathrm{Bi}, \mathrm{C}$, and $\mathrm{Al}_{2} \mathrm{O}_{3}$, Journal of the Optical Society of America 65(6), 1975, pp. 742 -744, DOI: 10.1364/JOSA.65.000742.

[18] Johnson P.B., Christy R.W., Optical constants of the noble metals, Physical Review B 6(12), 1972, pp. 4370-4379, DOI: 10.1103/PhysRevB.6.4370.

[19] Olmon R.L., Slovick B., Johnson T.W., Shelton D., Oh S.H., Boreman G.D., Raschke M.B., Optical dielectric function of gold, Physical Review B 86(23), 2012, article 235147, DOI: 10.1103/PhysRevB. $\underline{86.235147 .}$.

[20] Werner W.S.M., Glantschnig K., Ambrosch-Draxl C., Optical constants and inelastic electron -scattering data for 17 elemental metals, Journal of Physical and Chemical Reference Data 38(4), 2009, pp. 1013-1092, DOI: $\underline{10.1063 / 1.3243762 .}$

[21] Stahrenberg K., Herrmann Th., Wilmers K., Esser N., Richter W., Lee M.J.G., Optical properties of copper and silver in the energy range 2.5-9.0 eV, Physical Review B 64(11), 2001, article 115111, DOI: 10.1103/PhysRevB.64.115111.

[22] Yang H.U., D’Archangel J., Sundheimer M.L., Tucker E., Boreman G.D., Raschke M.B., Optical dielectric function of silver, Physical Review B 91(23), 2015, article 235137, DOI: $\underline{10.1103 / \text { Phys }}$ RevB.91.235137.

[23] Wu Y., Zhang C., Estakhri N.M., Zhao Y., Kim J., Zhang M., Liu X.-X., Pribil G.K., Alù A., Sнгн C.-K., Li X., Intrinsic optical properties and enhanced plasmonic response of epitaxial silver, Advanced Materials 26(35), 2014, pp. 6106-6110, DOI: 10.1002/adma.201401474.

[24] Gao L., Lemarchand F., Lequime M., Comparison of different dispersion models for single layer optical thin film index determination, Thin Solid Films 520(1), 2011, pp. 501-509, DOI: 10.1016 / j.tsf.2011.07.028. 
[25] Yakubovsky D.I., Arsenin A.V., Stebunov Yu.V., Fedyanin D.Yu., Volkov V.S., Optical constants and structural properties of thin gold films, Optics Express 25(21), 2017, pp. 25574-25587, DOI: $10.1364 /$ OE.25.025574.

[26] Palik E.D., Handbook of Optical Constants of Solids, Academic, San Diego, 1998.

[27] Rakić A.D., Duurišić A.B., Elazar J.M., Majewski M.L., Optical properties of metallic films for vertical-cavity optoelectronic devices, Applied Optics 37(22), 1998, pp. 5271-5283, DOI: 10.1364 / AO.37.005271.

[28] TREACY M.M.J., Dynamical diffraction explanation of the anomalous transmission of light through metallic gratings, Physical Review B 66(19), 2002, article 195105, DOI: 10.1103/PhysRevB.66.195105.

[29] RAKIĆ A.D., Algorithm for the determination of intrinsic optical constants of metal films: application to aluminum, Applied Optics 34(22), 1995, pp. 4755-4767, DOI: 10.1364/AO.34.004755.

Received October 31, 2018 in revised form April 16, 2019 